\title{
Expresión de proteínas del choque térmico H SP72 y HSP73 en casos colombianos de Linfoma de Hodgkin positivos y negativos para virus de Epstein Barr
}

\author{
Sandra M Q uijano G ${ }^{1 a}$, Carlos Saavedra A², \\ María Mercedes Bravo $H^{1 b}$, Susana Fiorentino ${ }^{3 c}$, \\ O scar O rozco D ${ }^{1 *}$.
}

\section{Expression of heat shock proteins HSP72 and HSP73 in Epstein-Barr positive or negative Hodgkin lymphoma}

Background: The expression of heat shock proteins (HSP70) in tumor cells or virus infected cells is important for the induction of specific cellular immune response. They are implicated in transport of immunodominants peptides in the endoplasmic reticulum, activation of antigen presenting cells and cross priming of CD8 T cells. AIM: To analyze the expression of HSP70 protein in its constitutive (HSP73) and inducible forms (HSP72) in Hodgkin's lymphoma (HL), infected or not by Epstein Barr virus (EBV) and to assess its relationship with pathological subtype, clinical stages and treatment response. Material and methods: The analysis of HSP73 and HSP72 was done by immunoperoxidase on routinely processed paraffin sections with prior antigen retrieval. Results: Sixty seven cases were studied. The expression of HSP73 and HSP72 was detected in 19.4 and $17.9 \%$ of samples respectively. The infiltrating lymphocytes expressed HSP72 in 58\% of cases. The pathological subtypes with the higher expression in lymphocytes were mixed cellularity and nodular sclerosis. No differences in HSP70 expression were observed, according to clinical stage, treatment response or the presence of EBV. Conclusions: The expression of HSP72 on lymphocytes suggests that this protein plays an important role in the induction and amplification of antitumor immune response (Rev Méd Chile 2003; 131: 1375-81).

(Key Words: Heat-Shock proteins 70; Herpes virus 4, human; Hodgkin's disease)

Recibido el 10 de diciembre, 2002. Aceptado en versión corregida el 7 de octubre, 2003. Trabajo financiado por Instituto Nacional de Cancerología. E.S.E. Bogotá, Colombia. Subproyecto de inversión 4103038-17.

${ }^{1}$ Laboratorio de Inmunología. Instituto Nacional de Cancerología. Bogotá, Colombia. ${ }^{2} \mathrm{Gru}$ po de Patología. Instituto Nacional de Cancerología. Bogotá, Colombia. ${ }^{3}$ Laboratorio de Inmunobiología. Pontificia Universidad Javeriana. Bogotá, Colombia.

aBacterióloga. MSc; b Microbióloga. MSc; ${ }^{C B}$ acterióloga PhD; *In memoriam.

Correspondencia a: María Mercedes Bravo. Grupo de Inmunología, Instituto Nacional de Cancerología. Calle 1a. № 9-85, Santafé de Bogotá, Colombia, Sudaménica. Fax: 57 (1) 3341360. Teléfono: 57 (1) 3340959. E mail: mbravo@incancerologia.gov.co 
Las as proteínas del choque térmico (HSPs), son moléculas que se expresan en una célula en respuesta a diferentes estímulos o condiciones de estrés ${ }^{1}$ y modulan procesos metabólicos importantes necesarios para el mantenimiento del equilibrio celular ${ }^{2}$. Su función como chaperonas moleculares durante el plegamiento, ensamblaje y traslocación de proteínas es esencial en organismos procariotes y eucariotes ${ }^{1}$. Las HSPs se han clasificado en cuatro familias denominadas HSP90, HSP70, HSP60 y HSPs pequeñas de acuerdo a su estructura, secuencia de aminoácidos, peso molecular y su grado de homología ${ }^{1}$.

Dentro de la familia HSP70 se encuentra la HSP73 que se ubica en el citosol y es expresada constitutivamente, tiene $90 \%$ de homología con la HSP72 la cual es inducible bajo condiciones de estrés $^{3}$. Se ha descrito que la expresión de HSP72 en células tumorales o células infectadas por virus es importante para la generación de respuesta inmune celular específica ${ }^{4-6}$. HSP72 se expresa en la membrana plasmática de células tumorales de diferentes tipos de cáncer ${ }^{7}$ y estimula de forma específica la respuesta inmune. Estas observaciones sugieren que esta proteína modula una vigilancia inmune que activa linfocitos $\mathrm{T}$, proporciona una línea de defensa adicional contra la infección y transformación maligna ${ }^{5}$ y aumenta la sensibilidad a lisis mediada por células NK efectoras ${ }^{4}$. Adicionalmente, se ha evaluado la expresión de HSP72 relacionada con infecciones virales. Se ha descrito un incremento en la expresión de HSP72 en linfocitos B transformados por el virus de Epstein Barr (EBV), dependiente de la presencia del virus ${ }^{8}$. Sin embargo, en estos estudios no son claras las diferencias en la expresión de HSP72 en células infectadas y no infectadas, la cual puede estar alterada como consecuencia del estrés dado por la infección viral o por la transformación neoplásica9.

EBV ha sido propuesto como posible factor etiológico en el desarrollo y patogénesis de Linfoma de Hodgkin $(\mathrm{LH})$, ya que en 40 a $100 \%$ de los LH es posible detectar DNA y RNA virales en las células tumorales de Reed-Sternberg $(\mathrm{RS})^{10-17}$. El $\mathrm{LH}$ es un tumor frecuente en el Instituto Nacional de Cancerología (INC) en Bogotá, Colombia, ya que representa $24,3 \%$ del total de los linfomas diagnosticados y tratados en esta institución ${ }^{18}$.
El objetivo de este estudio fue analizar la expresión de HSP70 en su forma constitutiva (HSP73) e inducible (HSP72) en los casos de LH positivos y negativos para EBV y relacionar su presencia con los diferentes subtipos histológicos, los estadios clínicos tumorales y la respuesta al tratamiento de los pacientes.

\section{MATERIALES Y MÉTODOS}

Pacientes. Se incluyeron 67 pacientes con LH que asistieron a consulta al INC en Bogotá entre agosto de 1994 y diciembre de 1998. Todos los pacientes recibieron tratamiento de quimioterapia convencional. Los datos clinicopatológicos y de seguimiento fueron obtenidos de sus historias clínicas. El pronóstico fue determinado por el intervalo libre de enfermedad y la supervivencia después del tratamiento. Este estudio fue aprobado por el comité de ética y el comité científico del INC.

Muestras. Se obtuvieron biopsias de ganglios linfáticos de los 67 pacientes en el momento del diagnóstico. Todos los casos fueron clasificados histológicamente siguiendo los criterios de la Organización Mundial de la Salud $(\mathrm{OMS})^{19}$. Las biopsias fueron incluidas en parafina y se usaron para la detección de transcritos de EBV por medio de hibridación in situ y para el análisis de la expresión de LMP-1 de EBV, HSP73 y HSP72 por inmunoperoxidasa.

Anticuerpos. Se utilizaron anticuerpos policlonales producidos en cabra: HSP72 (inducible) (Santacruz K20) y HSP73 (constitutiva) (Santacruz K19). Como marcador control positivo de las células tumorales se empleó el anticuerpo monoclonal CD30 (Dako M0723) y para el infiltrado se empleó el anticuerpo monoclonal CD45 (Dako M0701) y se analizó la presencia de linfocitos $\mathrm{T}$ con el anticuerpo CD3 (Dako M756). Se utilizó el anticuerpo anti-LMP-1 (Dako M0897). Como control positivo de HSP72 se utilizó la línea celular K562 sometida a tratamiento de choque térmico ${ }^{20}$.

Técnica de desenmascaramiento de antígeno. Para el análisis de la expresión de HSP73, HSP72, CD30 y CD45 se utilizó una solución de desenmascaramiento de antígeno (Dako S3308). Los tejidos se deparafinizaron e hidrataron y posterior- 
mente se sumergieron en esta solución precalentada a $95^{\circ} \mathrm{C}$ durante $40 \mathrm{~min}$. Luego se procedió con la técnica de inmunoperoxidasa.

Técnica inmunohistoquímica. En los cortes histológicos se realizó la técnica de inmunoperoxidasa ABC, utilizando el kit VECTASTAIN ABC (Vector PK-4000). La peroxidasa endógena se bloqueó con una solución de metanol-peróxido durante 30 min, después se incubó con suero normal de caballo, $20 \mathrm{~min}$. Posteriormente se adicionaron los anticuerpos primarios durante $1 \mathrm{~h}$ a temperatura ambiente, luego se incubó con el anticuerpo secundario (anti-IgG de cabra o de ratón marcado con biotina) durante $40 \mathrm{~min}$ y finalmente se incubó con estreptavidina-biotina-peroxidasa durante $1 \mathrm{~h}$. Para el revelado se empleó como sustrato Diaminobencidina. La contratinción se hizo con hematoxilina de Mayer. La lectura de los resultados fenotípicos se realizó evaluando positividad o negatividad de las células tumorales y de la población acompañante. Los casos se consideraron positivos si más de $10 \%$ de las células analizadas en cada caso mostraban expresión positiva en membrana o en citoplasma.

Hibridación in situ. Se utilizó el kit de hibridización (Dako K5201) y la sonda complementaria a los transcritos RNA de EBERs de EBV marcada con fluoresceína (Dako Y5200). Los tejidos se sometieron a tratamiento con proteinasa $\mathrm{K}$. La sonda anti-EBERs marcada con fluoresceína se incubó durante $1 \mathrm{~h} \mathrm{y}$ media a $55^{\circ} \mathrm{C}$ y posteriormente con el conjugado antifluoresceína marcado con fosfatasa alcalina durante 30 min. El revelado se hizo con una solución de NBT y BCIP y la contratinción con eosina.

Estadística. El paquete estadístico SPSS versión 9.0 fue empleado para el análisis estadístico de los datos. Se utilizó la prueba $\chi^{2}$ para comparar las frecuencias de expresión de las HSPs en relación con las variables clinicopatológicas analizadas. Se empleó la prueba exacta de Fisher cuando fue necesario.

\section{Resultados}

Se incluyeron en el estudio 67 casos, $28,5 \%$ de los casos eran niños de 0 a 15 años, 58,2\% eran adultos jóvenes de 16 a 49 años y 13,4\% eran adultos mayores de 50 años. El 71,64\% eran de sexo masculino (48 casos) y $28,36 \%$ de sexo femenino (19 casos). Los casos se ubicaron en los siguientes estadios clínicos: 16 casos en estadio I (23,88\%), 18 casos en estadio II (26,87\%), 22 casos en estadio III (32,84\%), 9 casos en estadio IV $(13,43 \%)$ y 2 casos no determinados (2,99\%). Se encontró un predominio del subtipo histológico de esclerosis nodular (EN) en todos los grupos de edad y el grupo que mostró mayor número de casos de celularidad mixta (CM) fue el de mayores de 50 años.

En cuanto a la respuesta al tratamiento, el 86,57\% (58 casos) entró en remisión completa; el 20,9\% (14 casos) presentó una o más recaídas y 8,96\% (6 casos) falleció. El promedio de sobrevida total (supervivencia acumulada) para los casos estudiados fue de 54,82 meses.

Se detectó expresión de CD30 en 94\% de los casos (63/67) a nivel de la célula tumoral de RS (Figura 1A). El antígeno común leucocitario CD45 fue positivo en $100 \%$ de los casos a nivel del infiltrado no neoplásico (Figura 1B). Igualmente, el infiltrado era en su mayoría de linfocitos TCD3 positivos en $100 \%$ de los casos.

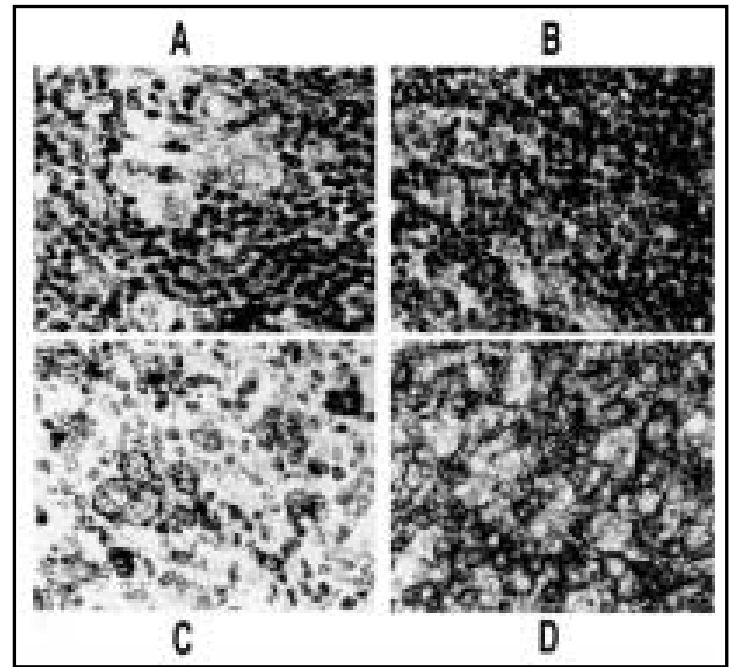

Figura 1. Caso de linfoma de Hodgkin donde se observa la expresión de CD30 en células tumorales (A) y de CD45 en infiltrado (B). En C y D se observan células tumorales acompañadas de linfocitos positivos para HSP72. Técnica inmunoperoxidasa. Aumento 40X. 
Infección con EBV en casos de LH. Se encontró un alto porcentaje de infección por EBV (67\% positivos para EBERs y $56,7 \%$ de expresión para la proteína oncogénica LMP-1), con alta prevalencia en los subtipos histológicos de EN y $\mathrm{CM}$ y en todos los grupos de edad (Tabla 1).

Los pacientes positivos para EBV presentaron una mejor respuesta al tratamiento reflejada en una menor tendencia a presentar recaídas en el tiempo en comparación con los no infectados (EBER: P LR test $=0,0005$; LMP-1: P LR test $=0,003$ ) o no con EBV (EBER: P LR test=0,3; LMP-1: P LR test $=0,22$ ).

Expresión de HSPs en LH. Se observó expresión de HSP73 y HSP72 tanto a nivel de la célula tumoral como a nivel del infiltrado linfocitario.

HSP73 se encontró expresada a nivel de la célula tumoral en 19,4\% de los casos (13/67), para HSP72 esta expresión fue de 17,9\% (12/67). La reactividad para ambas proteínas se presentó a nivel de citoplasma y membrana. No se encontró asociación significativa entre la expresión de HSP73 y de HSP72 en tumor con la presencia de transcritos EBER $(p=0,4)$ o con la expresión de la proteína viral LMP-1 $(\mathrm{p}=0,31)$.

En 35,8\% de los casos (24/67) se observó expresión de HSP73 en linfocitos infiltrantes de tumor, a nivel de citoplasma con un patrón de reactividad difuso. HSP72 se detectó a nivel de citoplasma en el infiltrado linfocitario en un 58,2\% de los casos (39/67) (Figura 1C y 1D). Se encontró un alto porcentaje de expresión de HSP72 en linfocitos en $62,2 \%$ de casos positivos para EBER como en $50 \%$ de los casos negativos. En los casos positivos y negativos para LMP-1 también se observó un alto porcentaje de expresión, 65,8\% y $48,3 \%$ respectivamente, sin encontrarse diferencias significativas entre positivos y negativos para EBV.

Expresión de HSP73 y HSP72 en los subtipos histológicos. Según el tipo histológico, se observó expresión de HSP73 en linfocitos infiltrantes del tumor en $37,7 \%, 28,6 \%$ y $20 \%$ de los casos de EN, CM y rico en linfocitos (RL); y a nivel de la célula tumoral en $17 \%$ y $42,9 \%$ de los casos de EN y CM respectivamente; en el tipo $\mathrm{RL}$ no se observó expresión de HSP73 en célula tumoral; HSP72 se detectó en los linfocitos infiltrantes en 62,3\%, $42,9 \%$ y $40 \%$ de los casos de EN, CM, y RL; en la célula tumoral la expresión de HSP72 se observó en $13,2 \%, 42,9 \%$, y $20 \%$ de los casos de EN, CM y $\mathrm{RL}$ respectivamente. No se incluyó en este análisis el tipo histológico predominio linfocítico por el bajo número de casos. No se encontró asociación entre la expresión de HSP73 y HSP72 y los subtipos histológicos. Sin embargo, se observó alta expresión de HSP72 en linfocitos dentro de cada uno de ellos. El subtipo de CM mostró la mayor expresión de HSP72 y HSP73 en la célula tumoral. No se encontró asociación entre la expresión de HSP73 y HSP72 en linfocitos o en tumor y la respuesta al tratamiento o los estadios clínicos tumorales.

Tabla 1. Prevalencia de infección por EBV (hibridación in situ para EBER e inmunoperoxidasa para LM P-1)

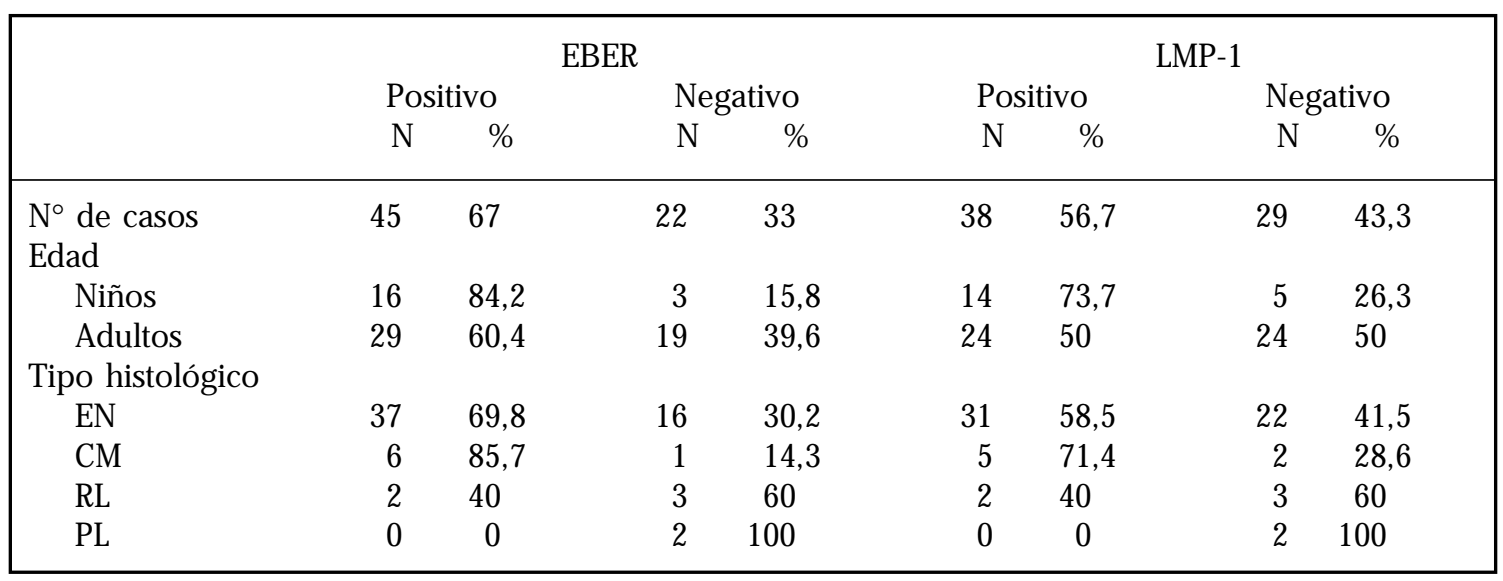

EN: esclerosis nodular. CM: celularidad mixta. RL: rico en linfocitos. PL: predominio linfocítico. 


\section{DISCUSIÓN}

El LH está constituido por un importante infiltrado inflamatorio que rodea la célula tumoral, que es reclutado en el tumor por la acción de citoquinas, la expresión de receptores de superficie celular y de péptidos de origen tumoral y viral en casos con presencia de EBV ${ }^{21}$. La célula tumoral infectada 0 no con EBV, secreta citoquinas y factores que inhiben la respuesta $\mathrm{T}$ específica de tumor y la función de células presentadoras de antígeno (CPA), facilitando de esta forma la persistencia del tumor en un ambiente rico en linfocitos ${ }^{22}$.

En todos los casos de $\mathrm{LH}$ analizados en este estudio se observó un infiltrado de linfocitos CD3+, con expresión de CD45 y con expresión de CD30 en la célula tumoral. Estos datos concuerdan con lo reportado en la literatura respecto a que en el tipo clásico de $\mathrm{LH}$ donde se presenta una respuesta inflamatoria mediada por linfocitos $\mathrm{T}^{23}$.

Las proteínas HSP73 y HSP72 se evidenciaron por inmunohistoquímica a nivel del infiltrado linfocitario y a nivel de la célula tumoral. El porcentaje de expresión fue diferente para cada proteína y bajo en la mayoría de casos en el tumor y no se encontró asociación entre la expresión de estas proteínas con la presencia de EBV en la célula tumoral.

La expresión de HSP72 en la célula tumoral es baja en comparación con la reportada por Takahashi en casos de LH en Japón ${ }^{24}$, donde encontró expresión de HSP72 en la célula tumoral en 85\% de los casos. La baja expresión en tumor observada en nuestro estudio podría ser debida a diferencias técnicas que incluyen el tipo de anticuerpos utilizados $^{24}$ y a la variabilidad geográfica, ya que nuestros casos de LH corresponden a un patrón epidemiológico de país en vía de desarrollo con predominio del subtipo de esclerosis nodular, con alta incidencia en todos los grupos de edad y con alta prevalencia de $\mathrm{EBV}^{25-26}$, el puede diferir a las características epidemiológicas encontradas en países desarrollados como Japón.

Hsu, en 1998, analizó la expresión de HSPs distintas a HSP72 y HSP73 (HSP89, HSP60 y HSP27) en casos de LH, encontrando abundante expresión de estas proteínas en la célula tumoral, atribuyendo este hecho a cambios en la tolerancia al calor (condición febril), a cambios en el metabolismo celular relacionados con la transformación maligna y como respuesta a citoquinas presentes en el microambiente tumoral las cuales pueden influir directa o indirectamente en la expresión de diferentes HSPs $^{27}$.

Adicionalmente, la baja expresión de HSP72 en el tumor podría ser un mecanismo de evasión de la respuesta inmune por el tumor y de resistencia a apoptosis. Se ha descrito que algunas HSPs están implicadas en la inducción o resistencia a la apoptosis dependiendo del origen celular $^{28}$. Sin embargo, en este estudio no encontramos asociación entre la expresión de estas proteínas con la respuesta de los pacientes al tratamiento.

De otro lado, la expresión de estas proteínas puede depender del tiempo de evolución de los pacientes. Teniendo en cuenta que los pacientes presentan distintos tiempos de evolución (2 meses a 2 años) y la expresión de estas proteínas podría perderse una vez que la transformación maligna inducida por EBV ha tomado lugar. Este mecanismo ha sido descrito al infectar linfocitos B in vitro con $\mathrm{EBV}^{8}$. Se ha demostraron que desde la unión del virus a su receptor en el linfocito $B$ se inducen HSP72 y HSP90. Los niveles se detectan a pocos minutos después de la exposición a EBV, con una expresión máxima a las $3 \mathrm{~h}$ y disminución a las 24 $\mathrm{h}$, lo que sugiere que su inducción es importante para el desarrollo del fenotipo transformado pero no para su mantenimiento ${ }^{8}$. Este hallazgo podría explicar, en parte, la baja expresión observada en nuestros casos, teniendo en cuenta que la célula tumoral de $\mathrm{LH}$ es de origen $\mathrm{B}$ y es rescatada de apoptosis por EBV, favoreciendo su sobrevida en un ambiente rico en linfocitos ${ }^{29}$.

A diferencia de otros estudios en los que se reporta la expresión de HSP72 en células tumorales pero no en células normales ${ }^{5}$, en nuestros casos observamos un alto porcentaje de expresión de HSP72 en el infiltrado linfocitario, en casos positivos y negativos para EBV y en todos los subtipos histológicos. Este resultado sugiere que HSP72 podría ser secretada por células normales y actuaría como una citoquina, que puede activar células presentadoras de antígeno (CPA) profesionales. Esta función de HSP72 como citoquina ha sido demostrada previamente; Basu en 2000, demostró que HSP72 es secretada y puede activar macrófagos y células dendríticas vía NFkB, induciéndolos a secretar cito- 
quinas y a expresar moléculas co-estimuladoras y de presentación antigénica ${ }^{30}$.

Asea, en 2000, también describió el papel de HSP72 como citoquina. Demostró que HSP72 exógena marcada con biotina se une con alta afinidad a la membrana plasmática de monocitos humanos, activa el flujo intracelular de calcio y la vía NFkB y sobrerregula la producción de citoquinas pro-inflamatorias en estas células ${ }^{31}$.

\section{REFERENCIAS}

1. SChlesinger M. Heat shock proteins. J Biol Chem 1990; 265: 12111-4.

2. Van Eden W, Van Der Zee R, Paul A. Do heat shock proteins control the balance of T-cell regulation in inflammatoty diseases? Immunology Today 1998; 19: 303-7.

3. Ciupitu A, Peterson M, O’Donnell C, Wimams K, Jindal S, KiesSLing R et al. Immunization with a lymphocytic choriomeningitis virus peptide in protective antiviral immunity and specific cytotoxic T lymphocytes. J Exp Med 1998; 187: 685-91.

4. $\mathrm{X}_{\mathrm{U}} \mathrm{Q}, \mathrm{W}_{\mathrm{ICK}} \mathrm{G}$. The role of heat shock proteins in protection and pathophysiology of the arterial wall. Mol Med Tod 1996; 2: 372-9.

5. Multhoff G, Botzier C, Wiesnet M, Muler E, Meier T, WiLmanNs W ET AL. A stress-inducible $72 \mathrm{kDa}$ heat-shock protein (HSP72) is expressed on the surface of human tumor cell, but not on normal cells. Int J Cancer 1995; 61: 272-9.

6. Heike M, Noll B, Meyer K. Heat shock proteinpeptide complexes for use in vaccines. J Leuk Biol 1996; 60: 153-8.

7. Yuquan W, Zhao $X$, Katriya $Y$, Fukata $H$, Teshiwara $\mathrm{K}$, UCHIDA A. Induction of autologous tumor killing by heat treatment of fresh human tumor cells: involvement of $\gamma \delta$ cells and heat shock protein 70. Cancer Res 1990; 36: 1104-10.

8. Cheung R, Dosch H. The growth transformation of human B cells involves superinduction of HSP72 and hsp90. Virology 1993; 193: 700-8.

9. Szekely L, Jiang W, Pokrouskaja K, Wiman K, Kiein G, RINGERTZ N. Reversible nucleolar traslocation of Epstein-Barr virus encoded EBNA-5 and hsp70 proteins after exposure to heat shock or cell density congestion. J Gen Virol 1995; 76: 2423-32.
Como conclusión podemos decir que HSP72 se puede expresar en células normales (linfocitos $\mathrm{T}$ infiltrantes de tumor) lo cual puede deberse a la activación inducida por estímulos derivados de la célula tumoral. Sería importante determinar si esta expresión resulta en la secreción de HSP72 y si al ser secretada es capaz de activar CPA profesionales como vía alterna de restauración de los mecanismos de procesamiento y presentación antigénica en tumores asociados a virus como LH.

10. Quintanila L, Gamboa A, Gamez I, Angeies A, Mohar A. Association of Epstein-Barr virus latent membrane protein and Hodgkin's disease in Mexico. Mol Pathol 1995; 8: 675-9.

11. Zhu Z, Hamilton S, Yan Q, Pallesen G. The association between Epstein-Barr virus and Chinese Hodgkin's Disease. Int J Cancer 1993; 55: 359-63.

12. Huh J, Park C, Juhng S, Kim C, Poppema S, Kim C. A pathologic study of Hodgkin Disease in Korea and its association with Epstein-Barr virus infection. Cancer 1996; 77: 949-55.

13. Chang K, Albujar P, Chen Y, Johnson R, Weiss L High prevalence of Epstein-Barr virus in the Reed-Sternberg cells of Hodgkin Disease ocurring in Peru. Blood 1993; 81: 496-501.

14. Ambinder R, Brownin P, Lorenzana I, Leventhal B, Cosenza H, ManN R et al. Epstein-Barr virus and childhood Hodgkin Disease in Honduras and the United States. Blood 1992; 81: 452-67.

15. HeRBST H. Epstein-Barr virus in Hodgkin's Disease. Sem Cancer Biol 1996; 7: 183-9.

16. Weiss L, Chen Y, Lu X, Shibata D. Epstein-Barr virus and Hodgkin's Disease. A correlative in situ hibridization and polymerase chain reaction study. Am J Pathol 1991; 139: 1259-65.

17. Preciado M, De Matteo E, Diez B, Menarguez J, GiNSTEIN S. Presence of Epstein-Barr virus and strain type assignment in Argentine childhood Hodgkin's Disease. Blood 1995; 86: 3922-9.

18. Registro Institucional de CÁnCER. Instituto Nacional de Cancerología. División de Epidemiología y Prevención. Grupo de Registro y Seguimiento. Colombia 1998.

19. HARRIS, N. Hodgkin's Lymphomas: Classification, Diagnosis and Grading. Sem in Hematology 1999; 36: 220-32. 
20. MulthofF G. Heat shock protein 72 (HSP72) a hyperthermia-inducible immunogenic determinant on leukemia K562 and Erwing's sarcoma cells. Int J Hyperthermia 1997; 13: 39-48.

21. Herbst H, RafF T, Sten H. Phenotypic modulation of Hodgkin and Reed-Sternberg cells by EpsteinBarr. J Pathol 1996; 179: 54-9.

22. Rooney C, Colton A, Heslop H. Control of virus induced lymphoproliferation: Epstein-Barr virus induced lymphoproliferation and host immunity. Mol Med Tod 1997; 3: 24-30.

23. Poppema S, Potters M, Emmens R, Visser L, Berg A. Immune reactions in classical Hodgkin's lymphomas. Sem in Haematol 1999; 36: 253-9.

24. Takahashi H, Fujita S, Shibata Y, Tsuda N, Okabe H. Expression of heat shock protein (HSP70) and EBV latent membrane protein 1 (LMP1) in ReedSternberg cells of Hodgkin's disease. Anal Cell Pathol 1996; 12: 71-83.

25. Glaser S, Lin R, Stewart S, Ambinder R, Jarret R, BRousset $P$ ET AL. Epstein-Barr virus associated Hodgkin's disease: epidemiologic characteristics in international data. Int J Cancer 1997; 70: 37582.

26. JARRET R, MACKENZIE J. Epstein-Barr virus and other candidate viruses in the pathogenesis of Hodgkin's disease. Sem in Haematol 1999; 36: 260-9.
27. Hsu PL, Hsu SM. Abundance of Heat Shock Proteins (hsp89, hsp60 and hsp27) in malignant cells of Hodgkin's disease. Cancer Research 1998; 58: 5507-13.

28. CREAGH E, CotTer S. Heat shock proteins-modulators of apoptosis in tumour cells. Leukemia 2000; 14: 1161-73.

29. KupPers R, Rajewsky K. The origin of Hodgkin and Reed-Sternberg cells in Hodgkin's disease. Annu Rev Immunol 1998; 16: 471-93.

30. Basu S, Binder R, Suto R, Anderson K, Srivastava P. Necrotic but not apoptotic cells death releases heat shock proteins, which deliver a partial maduration signal to dendritic cells and activate the NF-kB pathway. International Immunology 2000; 12: 1539-46.

31. Asea A, Kraeft S, Kurt E, Stevenson M, Chen L, FinBERg R ET AL. HSP72 stimulates cytokine production through a CD14 dependant pathway, demonstrating its dual role as a chaperone and cytokine. Nature America 2000; 6: 435-42.

Agradecimientos

Los autores agradecen la colaboración del Grupo de Patología del Instituto Nacional de Cancerología y al Grupo de Inmunobiología de la Pontificia Universidad Javeriana. 